Andr ogen and prost ate cancer: the rol e of pri mary andr ogen depr i vat i on ther apy in l ocal i zed prost at e cancer

\begin{tabular}{|l|l|}
\hline 著者 & $\begin{array}{l}\text { Nami ki M ki o, Ki t agawa Yasuhi de, M zokami } \\
\text { At sushi, Koh Ei t et su }\end{array}$ \\
\hline $\begin{array}{l}\text { j our nal or } \\
\text { publ i cat i on ti t l e }\end{array}$ & Journal of Men' s Heal th \\
\hline Vol une & 5 \\
\hline number & 4 \\
\hline page r ange & 333 -341 \\
\hline year & 2008 12-01 \\
\hline URL & ht t p: //hdl . handl e. net /2297/12650 \\
\hline
\end{tabular}




\section{Should patients with localized prostate cancer receive primary androgen deprivation therapy?}

Mikio Namiki*, Atsushi Mizokami and Hideyuki Akaza

\section{SUMMARY}

This Practice Point commentary discusses the study by Yao et al. in which primary androgen deprivation therapy (PADT) was compared with conservative treatment in elderly men with localized prostate cancer. Overall, PADT was associated with worse cancer-specific survival; however, in the subgroup of patients with poorly differentiated cancer, PADT was associated with improved cancer-specific survival compared with conservative management. Whereas the authors defined conservative treatment as no definitive treatment during the 180 days after diagnosis, many of these patients would have subsequently received definite treatments, including surgery or radiation therapy. The results, therefore, do not necessarily demonstrate inferiority of PADT to conservative treatment. Accurate evaluation of the efficacy of PADT is confounded by a number of factors, such as the type of ADT used. Efforts should be made to reduce the adverse effects of ADT, because a high proportion of patients actively choose to receive PADT.

Keywords: androgen deprivation therapy, conservative treatment, localized prostate cancer, overall survival, prostate-cancer-specific survival 


\section{COMMENTARY}

In an article published in The Journal of the American Medical Association, Yao et al. ${ }^{1}$ have investigated prostate-cancer-specific and overall survival in elderly men (greater than or equal to 66 years) with localized prostate cancer, comparing patients treated with primary androgen deprivation therapy (PADT; started within 180 days after diagnosis, with no surgery or radiation therapy given during this period) with those who received conservative treatment (no definitive treatment during this period). Statistical analysis was performed using instrumental variable analysis to control for both measured and unmeasured confounding variables. Of a total of 19,271 patients with localized prostate cancer, $41 \%$ received PADT and the remainder was managed conservatively. During the follow-up period, 1,560 patients died of prostate cancer and 11,045 deaths from all causes were reported. PADT was associated with lower prostate-cancer-specific survival and no change in overall survival compared with conservative management. For the subgroup of patients with poorly differentiated cancer, PADT was associated with improved prostate-cancer-specific survival, and a borderline improvement in overall survival. On the basis of the above results, the authors concluded that PADT was not associated with improved survival among the 
majority of elderly men with localized prostate cancer in comparison with conservative management.

The role of PADT in localized prostate cancer has not been well defined. Data on the current treatment of prostate cancer in Japan show that PADT is chosen for localized prostate cancer in an extremely high proportion of cases ${ }^{2}$. The US Cancer of the Prostate Strategic Urologic Research Endeavour (CaPSURE) has also shown increasing use of PADT for localized prostate cancer in recent years. Concern is growing, therefore, that PADT is being applied without clear evidence to support its use, because there have been many papers which showed ADT-associated morbidities.

Although the authors emphasized the inferiority of PADT in the treatment of localized prostate cancer, this conclusion has some limitations. The authors defined the PADT group as patients who received PADT during the 180 days after diagnosis, and the conservative treatment group as those who did not receive surgery, radiation therapy, or ADT during this period. A large number of patients in the latter group, however, might have received definitive treatments, including radical prostatectomy or radiation therapy, after 180 days. Indeed, one study has shown that approximately half of patients managed by watchful waiting received such treatments within 5 years. ${ }^{3}$ The results indicating that the PADT group had lower prostate-cancer-specific survival and similar 
overall survival compared with the conservative management group do not, therefore, necessarily demonstrate inferiority of PADT to conservative treatment, although it is unknown what percentage of the PADT group received definitive treatment after 180 days

The authors explained the poor cancer-specific survival of the subgroup of patients with moderately differentiated cancer who received PADT by suggesting that in these patients PADT might result in overgrowth of more-rapidly growing malignant clones. Unfortunately, such a hypothesis is unlikely to be tested in the clinical setting. Kitagawa et al. ${ }^{4}$ investigated the clinical and pathological effects of ADT using tissue specimens from patients treated with radical prostatectomy after neoadjuvant ADT. More than $40 \%$ of specimens showed complete histological cure (no cancer cells or complete apoptosis) or near cure (more than half of whole cancer cells apoptotic). In addition, the recurrence-free survival for patients with histologically cured specimens was $100 \%$. These results support the suggestion of long-term control or cure by PADT alone in some cases of localized prostate cancer. ${ }^{5,6}$ The present study should stress that the PADT-treated subgroup with poorly differentiated cancer demonstrated improved prostate-cancer-specific survival.

Currently, little high-quality evidence regarding comparative efficacy and 
adverse effects is available to guide patients selecting treatments for localized prostate cancer. ${ }^{7}$ Accurate evaluation of the efficacy of PADT is especially difficult, because many factors, such as type of ADT (i.e. combined androgen blockade, castration monotherapy or antiandrogen therapy), duration of ADT, and ethnicity, affect the outcome of therapy. Most patients, therefore, select a particular treatment on the basis of their own feelings. Many factors which contain not only efficacy and adverse effects, but also an anxiety or psychological burden about their treatments may be involved in their decision-makings. . Although clinical practice guidelines and most urologists do not recommend PADT as treatment for localized prostate cancer, many patients actually select PADT. We should consider why so many patients select PADT even after accurate, comprehensive explanations to aid in treatment decision-making by their attending physicians. Medical treatment, such as PADT, is probably more acceptable to some patients than more-invasive treatment, such as surgery In addition, their attending physicians themselves acquiesce to patients’ wishes, perhaps because these physicians have previously seen successful outcomes with PADT.. The Prostate Cancer Outcomes Study $^{8}$ of adverse effects of treatment and patient satisfaction yielded interesting results; satisfaction was higher in men who received early intervention, including PADT, than in those who received watchful waiting. In addition, the study showed that most patients 
who have received a particular treatment would make the same treatment selection again, no matter what treatment is the best. Therefore, what is important is how adequately the physicians are able to monitor and diminish potential consequences associated with each treatment

\section{References}

1 Lu-Yao GL et al. (2008) Survival following primary androgen deprivation therapy among men with localized prostate cancer. JAMA 300: 173-181

2 Cancer Registration Committee of Japanese Urological Association (2005)

Clinicopathological statistics on registered prostate cancer patients in Japan: 2000 report from the Japanese Urological Association. Int J Urol 12: 46-61

3 Meng MV et al. (2003) Predictors of treatment after initial surveillance in men with prostate cancer: results from CaPSURE. J Urol 170: 2279-2283

4 Kitagawa Y et al. (2003) Pathological effects of neoadjuvant hormonal therapy help predict progression of prostate cancer after radical prostatectomy. Int J Urol 10: $377-382$

5 Labrie F et al. (2002) Can combined androgen blockade provide long-term control or possible cure of localized prostate cancer? Urology 60: 115-119

$6 \quad$ Ueno S et al. (2006) Efficacy of primary hormonal therapy for patients with localized and locally advanced prostate cancer: a retrospective multicenter study. Int $J$ Urol 13: $1494-1500$

$7 \quad$ Wilt TJ et al. (2008) Systematic review: comparative effectiveness and harms 
of treatments for clinically localized prostate cancer. Ann Intern Med 148: 435-448 8 Hoffman RM et al. (2003) Patient satisfaction with treatment decisions for clinically localized prostate carcinoma: results from the Prostate Cancer Outcomes Study. Cancer 97: 1653-1662

M Namiki is Professor and Chairman, and A Mizokami is Associate Professor in the Department of Integrative Cancer Therapy and Urology, Kanazawa University Graduate School of Medical Science, Kanazawa, Ishikawa; H Akaza is Professor and Chairman in the Department of Urology, Institute of Clinical Medicine, University of Tsukuba, Tsukuba, Ibaraki, Japan.

\section{Competing interests}

The authors declared no competing interests.

\section{Correspondence}

*Department of Integrative Cancer Therapy and Urology

Kanazawa University Graduate School of Medical Science

13-1 Takaramachi

Kanazawa City

Ishikawa 920-8640

Japan

namiki1@kenroku.kanazawa-u.ac.jp

Received 5 September 2008

Accepted XXX

doi 


\section{Practice point}

Although this study found worse long-term cancer-specific survival with primary

androgen deprivation therapy (PADT) than with conservative treatment, the results do not necessarily indicate inferiority of PADT because of the confounding effect of subsequent definitive treatment. What is important is how adequately the physicians are able to monitor and diminish adverse effects of each treatment.

NAMIKI ET AL. XXX 2008 VOL 5 NO X 